\title{
ALEXANDER'S SUBBASE LEMMA
}

\author{
S. SALBANY AND T. D. TODOROV
}

(Communicated by James E. West)

This note provides a very simple proof of Alexander's subbase lemma, from the point of view of Nonstandard Analysis. There is no direct appeal to Zorn's lemma or equivalent principle (as in [1]). This set theoretic principle is, of course, embodied in the construction of the nonstandard extension ${ }^{*} X$.

Notation and terminology is that of A. Hurd and P. Loeb [2].

Lemma (Alexander). Let $(X, T)$ be a topological space and $\mathscr{S}$ a subbase of closed sets. If every family of closed sets in $\mathscr{S}$ with the finite intersection property has nonempty intersection, then $(X, T)$ is compact.

Proof. Recall that $(X, T)$ is compact iff ${ }^{*} X=\bigcup_{x \in X} \mu(x)$ [2, Theorem (2.9), Chapter III] and that the monad of $x$ is $\mu(x)=\bigcap\left\{{ }^{*} G \mid x \in G, X-G \in \mathscr{S}\right\}$ [2, Proposition (1.4) of Chapter III]. Let $\alpha \in{ }^{*} X$. Consider $\mathscr{F}=\{F \mid F \in \mathscr{S}$, $\left.\alpha \in{ }^{*} F\right\}$. Then $\mathscr{F}$ has the finite intersection property and, by assumption, there is a point $x$ such that $x \in \bigcap\{F \mid F \in \mathscr{F}\}$. We show that $\alpha \in \mu(x)$ : if $x \in G$ and $X-G \in \mathscr{S}$, then $\alpha \notin^{*}(X-G)$, by our choice of $x$, hence $\alpha \in{ }^{*} G$, as required.

\section{REFERENCES}

1. J. L. Kelley, General topology, Van Nostrand, New York, 1955.

2. A. E. Hurd and P. A. Loeb, An introduction to nonstandard real analysis, Academic Press, 1985.

Department of Mathematics, University of Zimbabwe, Harare, Zimbabwe

International School for Advanced Studies (SISSA/ISAS), 34014 Trieste, Italy

Received by the editors June 13, 1988.

1980 Mathematics Subject Classification (1985 Revision). Primary 03H05, 54J05, 54D30.

Key words and phrases. Nonstandard extension, Alexander's subbase lemma. 